\title{
Stupéfiants et conduite automobile - les actions réalisées en Belgique
}

\section{Drugs and driving - the Belgian experience}

\author{
Viviane MAES $^{*(1)}$, Nele SAMYN ${ }^{(2)}$, Michel WILLEKENS ${ }^{(3)}$, \\ Gert DE BOECK ${ }^{(2)}$, Alain VERSTRAETE(4)
}

(1) Département de Chimie Clinique - Toxicologie, Hôpital Académique - VUB - BRUXELLES

(2) Institut National de Criminalistique et de Criminologie - BRUXELLES

(3) Police Fédérale, DGJ/DJP, Service Central Drogues - BRUXELLES

(4) Laboratoire de Biologie Clinique - Toxicologie, Hôpital Universitaire - GENT

* Auteur à qui adresser la correspondance :Viviane MAES, Département de Chimie Clinique - Toxicologie, Hôpital Académique, VUB Laarbeeklaan 101 - B-1090 BRUXELLES - BELGIQUE

Tél : 3224775042 - Fax : 3224775047 - E-mail : viviane.maes@az.vub.ac.be

(Reçu le 8 avril 2003 ; accepté le 26 avril 2003)

\section{RÉSUMÉ}

En mars 1999 une nouvelle loi réprimant la conduite sous influence de drogues illicites a été introduite en Belgique. La procédure légale consiste en a) un test de comportement, $b$ ) un dépistage immunochimique pour 4 groupes de drogues dans les urines $c$ ) une confirmation par une analyse du plasma (GC-MS avec des seuils fixes). Sur une période de deux ans, l'analyse de 896 échantillons sanguins a révélé la présence de drogues au-dessus du seuil légal dans $85 \%$ des cas. Dans les $15 \%$ de cas "faux positifs" (test de comportement et test urinaire positif sans confirmation dans le plasma), une analyse rétrospective de l'alcool et de médicaments a été entreprise.

Dans 36,8 \% des cas positifs, seul le THC a été détecté. L'amphétamine et/ou la MDMA étaient présentes au-dessus du seuil dans $32,1 \%$ des cas positifs, et elles étaient combinées au THC dans 11,8\% des cas. La cocaïne et/ou la benzoylecgonine ont été retrouvées dans $18,0 \%$ et la morphine dans $1,4 \%$ des cas positifs.

Dans les cas "faux positifs", 37 \% étaient positifs pour l'alcool, et $14 \%$ contenaient des médicaments, surtout des benzodiazépines.

Dans notre série, l'abolition du seuil légal aurait réduit les "faux positifs" de 15 à $9 \%$. En considérant les cas positifs pour l'alcool, il n'y a plus que $7 \%$ de "faux positifs". Si les médicaments étaient également couverts par la loi, les "faux positifs" seraient réduits de $1 \%$.

\section{MOTS-CLÉS}

conduite automobile, stupéfiants, alcool, médicaments psychoactifs, législation.

\section{SUMMARY}

In March 1999 a new law prohibiting driving while impaired by illegal drugs was introduced in Belgium. The legal procedure consists of a) a field impairment test, b) a urine immunoassay for 4 drug groups and c) ultimate proof by plasma analysis (GC-MS with fixed cut-offs). Over about two years the analysis of 896 blood samples revealed the presence of illicit drug(s) above cut-off in $85 \%$ of the cases. For the $15 \%$ "false positives" (failed impairment test and positive urine assay without confirmation in plasma), retrospective analysis of alcohol and medication was performed.

In $36,8 \%$ of the legally positive cases, THC was the only analyte detected. Amphetamine and/or MDMA were present above cut-off in $32,1 \%$ and combined with THC in $11,8 \%$ of all positive samples. Cocaine and/or benzoylecgonine were found in $18,0 \%$ and morphine in 1,4\% of the legally positive cases. In the "false positive" samples $37 \%$ were alcohol-positive, while $14 \%$ contained psychoactive medicines, mostly benzodiazepines.

In the studied population the abolition of the legal cut-off values would have resulted in a decrease of "false positives" from $15 \%$ to $9 \%$. Considering the additional legally positive alcohol cases a further decrease to $7 \%$ is observed. Including medicinal drugs in the law would have reduced the number of "false positives" by a further $1 \%$.

\section{KEY-WORDS}

impaired driving, illicit drugs, alcohol, psychoactive medicines, legislation. 


\section{Introduction}

Une nouvelle loi portant sur la conduite sous l'influence de drogues illicites a été introduite en Belgique en mars 1999 (1). Les classes de drogues mentionnées dans la loi sont limitées aux amphétamines (amphétamine, MDMA, MDEA, MBDB), la cocaïne (benzoylecgonine, cocaïne), les opiacés (morphine) et le cannabis (tétrahydrocannabinol), qui sont des groupes de drogues pour lesquels il existait des tests d'urine sur le marché belge. Les fonctionnaires de police attendaient depuis longtemps une procédure qui permet de prouver que certains conducteurs étaient non seulement sous l'influence de l'alcool mais également sous l'influence d'autres substances susceptibles d'altérer la conduite.

La loi initiale de 1968 comprenait un article (art. 35) spécifiant l'interdiction de conduire en état d'ivresse ou dans un état analogue résultant de la consommation de drogues (que celles-ci soient licites ou illicites). Une autre loi sur le code de la route (A.R. 01/12/75 - article 8.3) stipule qu'un conducteur doit à tout moment être capable d'effectuer n'importe quelle manœuvre et posséder les capacités et les compétences nécessaires pour conduire. Cependant, aucune méthode détaillée de test n'étant définie, le fonctionnaire de police devait attendre une décision individuelle du Parquet pour chaque cas. Pour autant que les auteurs de ce travail aient pu le vérifier, personne n'a jamais été condamné pour avoir transgressé ces articles.

Les résultats des premières d'années d'application de la nouvelle loi ont été évalués et la validité de la nouvelle procédure a été analysée.

\section{Matériel et méthodes}

\section{La procédure légale}

\section{La batterie de tests standurdisés (Figure 1)}

Ces tests portent sur :

- Symptômes physiques : yeux, visage et "autres"

- Comportement

- Langage

- Démarche

- Autres signes

- Tests de répartition d'attention (Figure 2).

Ces tests standardisés sont repris dans une Circulaire du Collège des Procureurs-Généraux auprès des Cours d'Appel. La circulaire stipule que lors de son application, la batterie de tests constitue un tout et sera considérée comme positive lorsqu'elle aura été parcourue complètement et que plusieurs signes auront été constatés, dont au moins un dans les signes corporels et un dans les tests de répartition d'attention.
Si la batterie de tests standardisés ne permet pas d'établir clairement que le sujet "est sous influence", la procédure est arrêtée à ce stade.

\section{Le test d'urine}

Lors d'une mise en évidence de signes de diminution de la capacité de conduire, le fonctionnaire de police peut entamer la seconde partie de la procédure, à savoir le test d'urine. Si des sanitaires ne sont disponibles sur place, la personne doit être emmenée au poste de police pour permettre le recueil d'un échantillon d'urine. La loi stipule clairement que toutes les mesures nécessaires doivent être prises pour préserver l'intimité de la personne. D'autre part, la circulaire du Parquet concernant les actes de police indique également que la police doit prendre toutes les mesures requises afin d'éviter l'altération de l'échantillon d'urine.

Une rapide analyse immunochimique de l'urine est effectuée sur place. Théoriquement, l'agent de police a le choix entre un test monoparamétrique (nécessitant l'emploi d'une pipette) et un test multiparamétrique (nécessitant l'immersion d'une carte), mais en pratique la majorité utilise le test multiparamétrique. Le test "Dipro drug screen 5" (groupe VanDePutte, Boechout, Belgique) permet d'obtenir des résultats pour cinq produits : les cannabinoïdes ( $\mathrm{Cnb}$ ) (seuil de détection $50 \mathrm{ng} / \mathrm{ml}$ ), les opiacés (Opc) (seuil de détection $300 \mathrm{ng} / \mathrm{ml}$ ), la cocaïne (Coc) (seuil de détection 300 $\mathrm{ng} / \mathrm{ml}$ ), les méthamphétamines (seuil de détection 500 $\mathrm{ng} / \mathrm{ml}$ ) et les amphétamines (seuil de détection 1000 $\mathrm{ng} / \mathrm{ml}$ ) (Amph). Le test multiparamétrique a été évalué de manière détaillée dans le laboratoire et sur le terrain et a montré une excellente réactivité croisée pour la MDMA et la MDA (2).

\section{L'analyse de sang}

Si le test d'urine se révèle positif pour au moins une classe de drogues, un médecin est chargé de faire un prélèvement de sang d'un volume minimal de $15 \mathrm{ml}$, qui sera envoyé le plus rapidement possible à un laboratoire agréé. Les valeurs limites pour l'analyse du plasma sont également mentionnées dans les textes de loi: THC (tétrahydrocannabinol) $2 \mathrm{ng} / \mathrm{ml}$, morphine libre $20 \mathrm{ng} / \mathrm{ml}$, amphétamine, MDMA, MDEA, $\mathrm{MBDB}$, benzoylecgonine (BE) ou cocaïne $50 \mathrm{ng} / \mathrm{ml}$. Le laboratoire dispose de 15 jours après réception de la réquisition pour transmettre les résultats. Si les concentrations détectées dans le plasma dépassent les valeurs limites spécifiées dans la loi, et uniquement dans ce cas, le conducteur est considéré comme conduisant sous l'influence de drogue. Il doit être informé des résultats de l'analyse dans les 30 jours, et il dispose ensuite de 14 jours pour décider s'il souhaite une contre-expertise. 


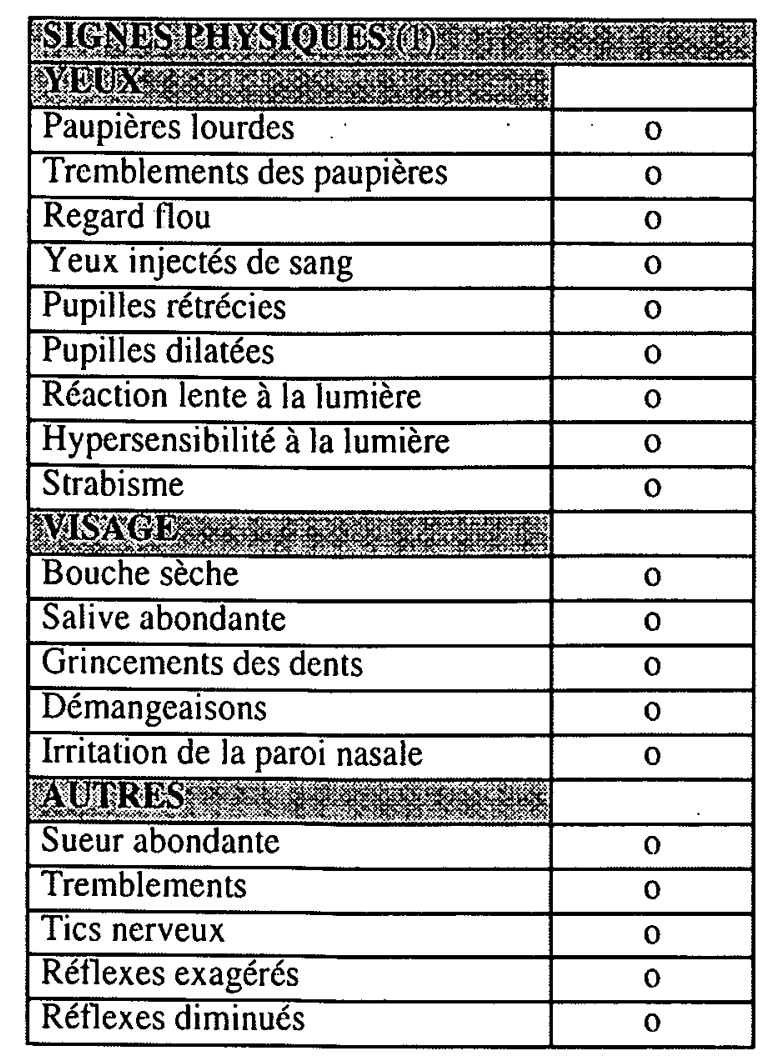

\begin{tabular}{|c|c|c|c|}
\hline \multicolumn{4}{|c|}{ 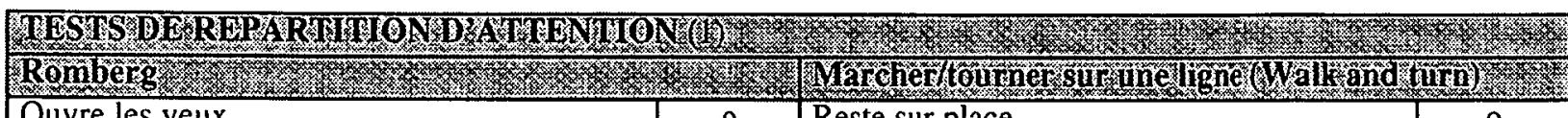 } \\
\hline & 0 & Reste sur place & 0 \\
\hline$<30^{\prime \prime}$ & 0 & Départ prématuré & 0 \\
\hline$>30^{\prime \prime}$ & 0 & S'arrête/hésite pendant l'exécution & 0 \\
\hline Vacille & $\overline{0}$ & Espace entre les pieds & 0 \\
\hline & & S'écarte de la ligne & 0 \\
\hline & & Lève les bras & 0 \\
\hline & & Trouble d'équilibre au tournant & 0 \\
\hline & & Mauvais nombre de pas & 0 \\
\hline & & Ne tourne pas correctement & 0 \\
\hline \multicolumn{2}{|c|}{ 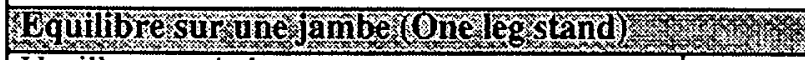 } & Dorgtspontes pers & Wrix \\
\hline Vacille en se balançant & 0 & Mauvaise main & 0 \\
\hline Utilise les bras & 0 & Mauvais endroit. & 0 \\
\hline Sautille & 0 & & \\
\hline Repose le pied levé & 0 & & \\
\hline Eprouve des difficultés à compter & 0 & & \\
\hline
\end{tabular}

(1) Marquer d'une croix ce qui convient. On peut soupçonner l'état d'influence sur la conduite d'une des substances mentionnées par la loi lorsque plusieurs cases ont été cochées, dont au moins une dans la partie «signes physiques » et une dans la partie « tests de répartition d'attention ».
Figure 1 : Formulaire à remplir ave les résultats de la batterie de tes standardisés.

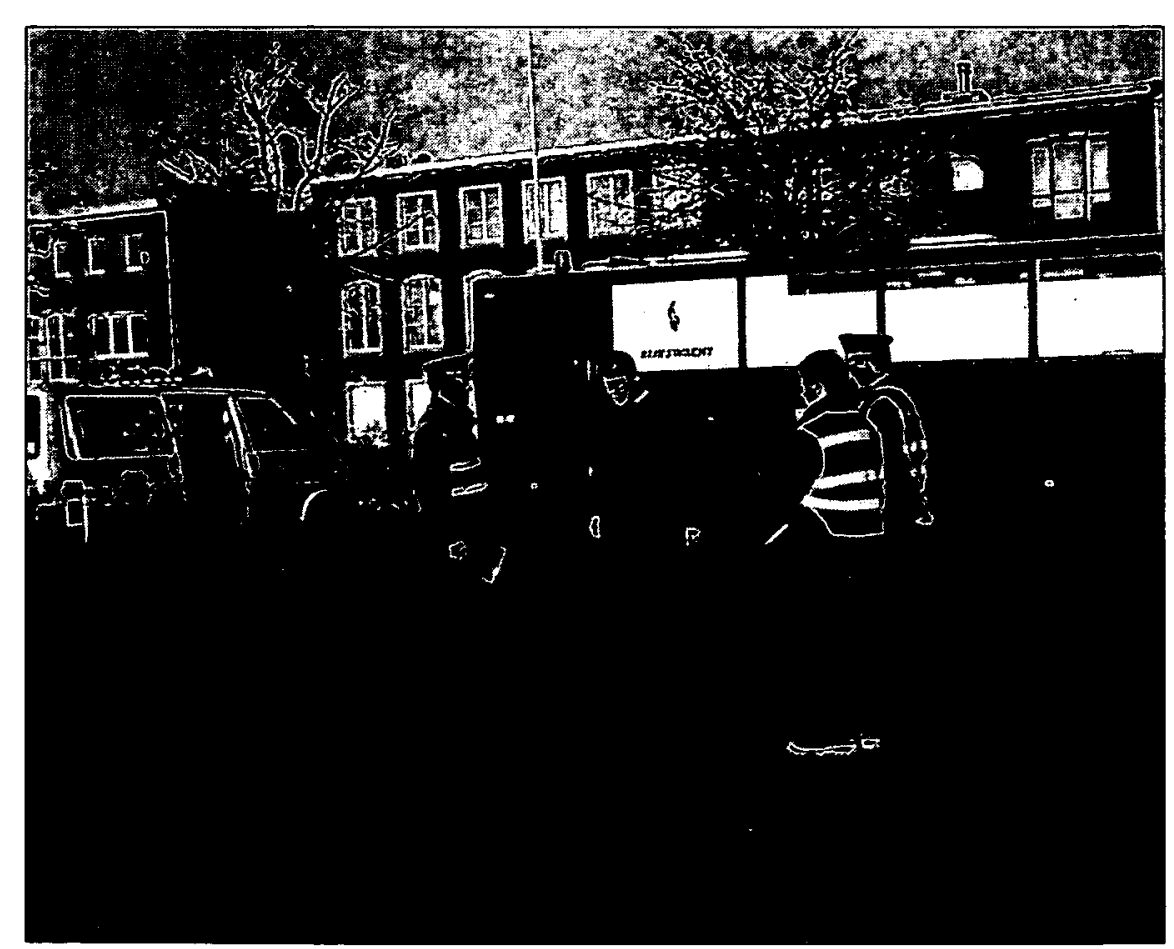

Figure 2 : Exemple d'un test psychomoteur issu de la "batterie de tests standardisés". En arrière plan se trouve un véhicule "bus bureau", où sera rédigé le procès-verbal si le conducteur s'avère positif aux tests physiques et au test urinaire.
Un conducteur peut refuser de se soumettre à la batterie de tests, au test d'urine ou au prélèvement sanguin. Ce refus est cependant punissable, la peine étant identique en cas de refus ou de résultat positif.

\section{Les sujets}

La population cible de cette étude sont des conducteurs arrêtés par la police belge dans la période 2000-2001, suspectés de conduite sous l'influence de drogues.

\section{Les prélèvements}

Dans la période de 2000-2001 l'Institut National pour Criminalistique et Criminologie (I.N.C.C.) a reçu 896 de prélèvements de plasma de cas de conduite présumée sous influence. Après les confirmations par GCMS, les plasmas ont été conservés à $-20^{\circ} \mathrm{C}$. Les prélèvements qui ne contenaient pas de drogues dans des concentrations supérieures aux limites légales, ont été testés rétrospectivement sur la présence d'alcool et de médicaments psychotropes. A titre de comparaison 
dans le temps, les mêmes analyses ont été effectuées sur un petit groupe de contrôle de 74 plasmas légalement positifs.

\section{Méthodes analytiques}

Les drogues cibles ont été analysées dans le plasma par des techniques d'extraction conventionnelles et par GC-MS utilisant des étalons internes deutérés ; les limites de quantification (LDQ) pour les différents composés satisfont largement aux valeurs limites stipulées dans la loi (3).

L'alcool a été analysé par une méthode GC directe (LDQ $0.05 \mathrm{~g} / \mathrm{L}$ ). La concentration plasmatique d'alcool est convertie en alcoolémie correspondante en divisant par un facteur de 1.14 (4). Les médicaments psychotropes ont été décelés et quantifiés par une méthode HPLC couplée à un détecteur à barette de diodes, couvrant les benzodiazépines, les antidépresseurs, certains neuroleptiques et analgésiques narcotiques.

\section{Résultats}

\section{L'implémentation de la loi}

\section{La batterie de tests standardisés}

Les premières séances de formation pour la Police Fédérale avaient déjà été organisées avant la publication de la loi. Environ 200 policiers de tout le pays, experts locaux en matière de drogues et enseignants (possédant une certaine expérience dans l'enseignement en matière de drogue) venant des écoles de police ont suivi une formation d'une semaine. La théorie a été mise en pratique à l'occasion d'un contrôle routier. Quelques erreurs mineures se sont manifestées au cours de ces exercices pratiques et le programme de formation a été immédiatement adapté. Une fois formés, ces policiers ont été chargés de transmettre leur savoir au reste du corps de police.

La plupart des contrôles sont concentrés sur les endroits où il est le plus probable de trouver des conducteurs positifs : à proximité des discothèques ou des "after-clubs". Une autre partie des contrôles est concentrée sur les routes et autoroutes venant des frontières nord du pays (personnes fréquentant les "coffeeshops"). La meilleure méthode semble être de poster sur la route un guetteur (policier expérimenté) qui effectue un premier filtrage. Le second filtre est la première étape de la procédure, c'est-à-dire la batterie de tests standardisés. Cette procédure a prouvé son efficacité : lors de plusieurs contrôles effectués un dimanche matin, deux conducteurs arrêtés sur trois ont été contrôlés positifs.

\section{Résultats des tests d'urine effectués sur le terrain}

Pendant la période 2000-2001, l' I.N.C.C. a enregistré 896 cas de "conduite sous l'influence de drogues". Sur base des résultats obtenus avec les tests d'urine réalisés sur le terrain, des échantillons de plasma correspondants ont été demandés pour confirmation d'un ou plusieurs analytes repris dans la loi. Dans 121 cas (13,5\% du total), l'échantillon d'urine n'a pas été fourni. Les résultats des contrôles effectués sur le terrain indiquent une fréquence élevée de consommateurs de cannabis et de produits stimulants dans cette population (tableau I). Il est assez fréquent de rencontrer des combinaisons de plusieurs drogues.

Tableau I : Tests d'urine positifs effectués sur le terrain. Au total, les cannabinoïdes étaient positifs dans 78,1\% des cas, les amphétamines dans 49,6\%, la cocaïne dans $20,2 \%$ et les opiacés dans 9,5\%.

\begin{tabular}{|l|cc|}
\hline Classes de drogues/Urine & Total (= 775) & \% \\
\hline Cnb & 304 & 39,2 \\
Amph/Cnb & 160 & 20,6 \\
Amph & 100 & 12,9 \\
Amph/Cnb/Coc & 54 & 7,0 \\
Amph/Coc & 36 & 4,6 \\
Cnb/Coc & 30 & 3,9 \\
Cnb/Opc & 21 & 2,7 \\
Amph/Cnb/Opc & 20 & 2,6 \\
Coc & 11 & 1,4 \\
Cnb/Coc/Opc & 10 & 1,3 \\
Opc & 9 & 1,2 \\
Amph/Cnb/Coc/Opc & 6 & 0,8 \\
Amph/Coc/Opc & 5 & 0,6 \\
Coc/Opc & 5 & 0,6 \\
Amph/Opc & 4 & 0,5 \\
\hline
\end{tabular}

\section{Résultats des analyses de sang}

Des concentrations de drogues illicites supérieures à la valeur limite autorisée ont été identifiées dans 763 échantillons.

La figure 3 donne un relevé des différentes combinaisons de drogues illicites identifiées dans le plasma. Le tableau II illustre les médianes de concentration et la fourchette des analytes les plus fréquents dans les plasmas des conducteurs pénalisés.

Le cannabis et les amphétamines sont les principales drogues décelées dans ces échantillons. Dans le groupe de conducteurs positifs pour amphétamines, la consommation de MDMA (64\%) et d'amphétamine (34\%) était la plus importante; la MDEA ( $2 \%$ ) était rarement détectée et aucun cas de MBDB n'a été décelé.

\section{Les "faux positifs"}

Le bien-fondé de la nouvelle procédure légale destinée 
Annales de Toxicologie Analytique, vol. XV, $\mathrm{n}^{\circ}$ 2, 2003

Tableau II : Médiane et limites des concentrations $(\mathrm{ng} / \mathrm{ml})$ d'analytes cibles dans le plasma des conducteurs pénalisés.

\begin{tabular}{|c|c|c|c|c|c|}
\hline & THC & MDMA & Amph & BE & Morphine \\
\hline Cnb seul & $\begin{array}{l}\mathbf{7 , 8} \\
(2,0-97,0)\end{array}$ & & & & \\
\hline Cnb/Amph & $\begin{array}{l}\mathbf{4 , 4} \\
(2,0-27,5)\end{array}$ & $\begin{array}{l}\mathbf{2 7 9} \\
(27-1582)\end{array}$ & $\begin{array}{l}\mathbf{9 0} \\
(13-980)\end{array}$ & & \\
\hline Amph seul & & $\begin{array}{l}\mathbf{3 4 5} \\
(10-5003)\end{array}$ & $\begin{array}{l}147 \\
(14-2146)\end{array}$ & & \\
\hline Amph/Coc & & $\begin{array}{l}\mathbf{4 1 9} \\
(54-1570)\end{array}$ & $\begin{array}{l}99 \\
(24-3163)\end{array}$ & $\begin{array}{l}\mathbf{3 2 5} \\
(53->5000)\end{array}$ & \\
\hline Cocaïne seul & & & & $\begin{array}{l}448 \\
(66->5000)\end{array}$ & \\
\hline Coc/Opc & & & & $\begin{array}{l}\mathbf{7 7 8} \\
(95-2685)\end{array}$ & $\begin{array}{l}35 \\
(28-64)\end{array}$ \\
\hline Opc total & & & & & $\begin{array}{l}32 \\
(24-64)\end{array}$ \\
\hline
\end{tabular}

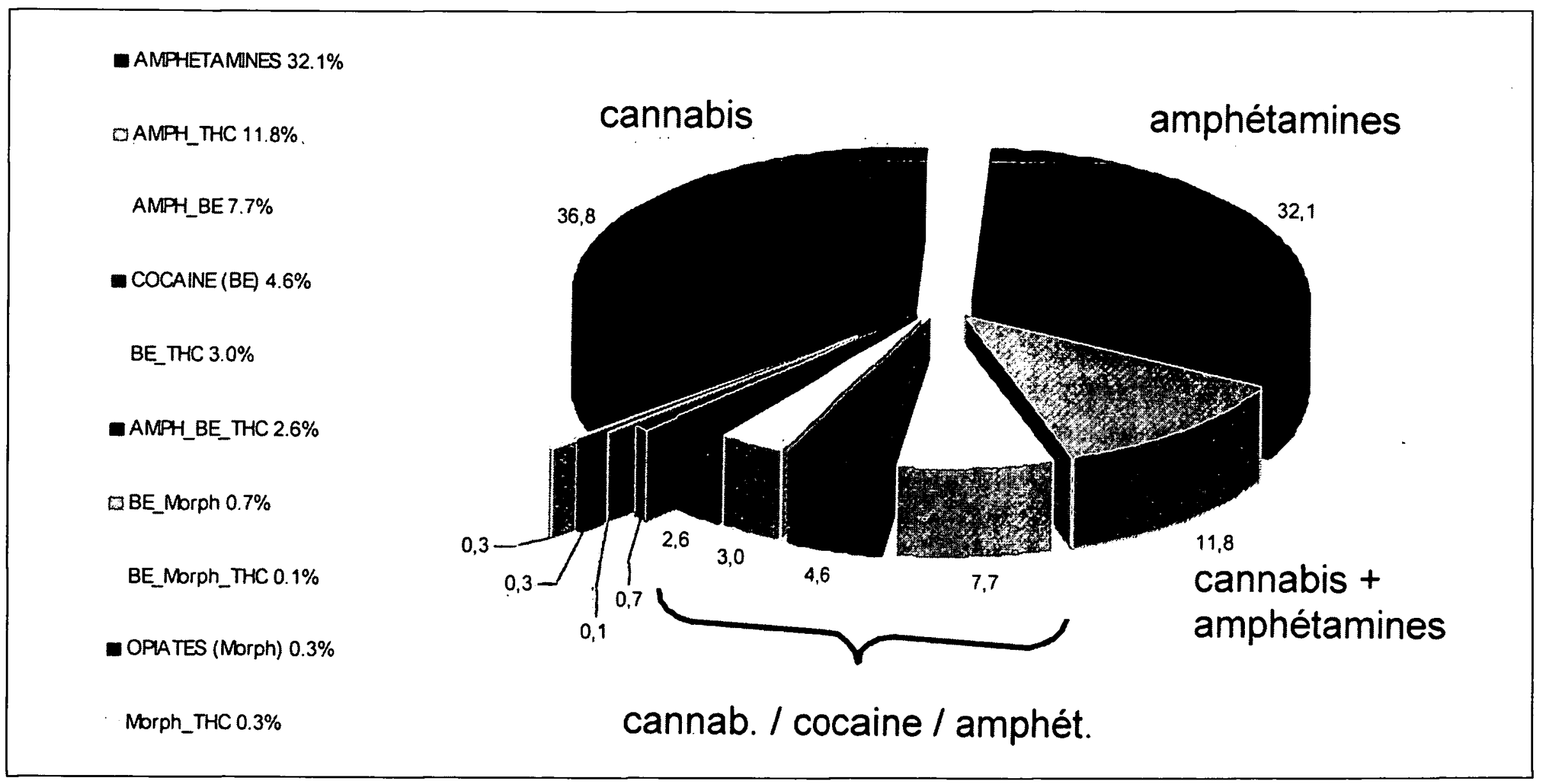

Figure 3 : Fréquence de différentes drogues illicites décelées dans les échantillons de plasma de conducteurs dont la capacité à conduire était diminuée.

à détecter les personnes conduisant sous l'influence de drogues illicites est prouvé par le nombre d'analyses de plasma confirmées positives. Pour la période 20002001, l'analyse de 896 échantillons sanguins révéla dans $85 \%$ des cas la présence d'une ou plusieurs drogues illicites en concentration supérieure à la valeur limite légale. Nous avons examiné les $15 \%$ de cas "faux positifs", c.-à-d. les cas où la batterie de tests standardisés s'est révélée positive et où le test urinaire s'est révélé positif pour au moins un groupe de drogues, mais où l'analyse du plasma n'a pas permis de déceler une concentration de drogue illicite supérieure à la valeur limite autorisée. Dans ces cas-là, la procédure légale pour conduite sous l'influence de drogues illicites a été menée à terme, mais le conducteur n'a subi aucune sanction, ni amende.

Pour les 133 cas ( $15 \%$ du total) où le plasma n'a pas révélé de drogues illicites supérieures à la limite légale, les données des tests d'urine et de plasma sont détaillées dans les tableaux III-VI. 
Tableau III : Résultats des tests d'urine réalisés sur le terrain pour les cas "faux positifs" (résultat positif pour le test d'urine, mais négatif pour l'analyse de sang). Au total, les cannabinoïdes étaient positifs dans $80,3 \%$ des cas, les amphétamines dans 20,8\%, la cocaïne dans $8,4 \%$ et les opiacés dans $17,5 \%$.

\begin{tabular}{|l|cc|}
\hline Classes de drogues/Urine & Total (=121) & \% \\
\hline Cnb & 74 & 61,2 \\
Cnb/Amph & 10 & 8,3 \\
Cnb/Opc & 7 & 5,8 \\
Cnb/Coc & 1 & 0,8 \\
Cnb/Opc/Amph & 2 & 1,7 \\
Cnb/Opc/Coc & 2 & 1,7 \\
Cnb/Amph/Coc & 1 & 0,8 \\
Amph & 10 & 8,3 \\
Amph/Coc & 2 & 1,7 \\
Opc & 8 & 6,6 \\
Opc/Coc & 2 & 1,7 \\
Coc & 2 & 1,7 \\
\hline
\end{tabular}

Tableau VI : Détail des résultats positifs pour les médicaments, avec les données correspondantes du screening urinaire d'alcool et des drogues illicites.
Tableau IV : Nombre de cas où les concentrations de drogues cibles illicites détectées dans le plasma étaient inférieures à la valeur limite légale.

\begin{tabular}{|lc|}
\hline Concentration dans le plasma < seuil & n \\
\hline THC $<2$ & 32 \\
Amphétamine ou MDMA $<50$ & 15 \\
Métabolite de cocaïne $<50$ & 10 \\
Morphine $<20$ & 3 \\
Total & 52 \\
\hline
\end{tabular}

Tableau V : Nombre d'alcoolémies positives combinées à la présence de drogues illicites en concentration inférieure à la valeur légale ("seuil").

\begin{tabular}{|l|ccc|}
\hline & $\mathbf{N}$ & Alcool $>\mathbf{0 , 5} \mathbf{g} / \mathbf{l}$ & Alcool $<\mathbf{0 , 5} \mathbf{g} /$ \\
\hline Alcool + crb<seuil & 12 & 9 & 3 \\
Alcool + amph<seuil & 5 & 4 & 1 \\
Alcool + coc/amph<seuil & 1 & 1 & 0 \\
Alcool + coc<seuil & 2 & 1 & 1 \\
Alcool + drogues $<$ seuil & 20 & 15 & 5 \\
\hline
\end{tabular}

\begin{tabular}{|c|c|c|c|}
\hline $\begin{array}{l}\text { Médicaments } \\
(\mu \mathrm{g} / \mathrm{l}) \text { (supra-thérapeutique) }\end{array}$ & $\begin{array}{l}\text { Alcool } \\
(\mathrm{g} / \mathrm{l})\end{array}$ & Test urine & $\begin{array}{c}\text { Drogue }<\text { seuil } \\
\text { plasma }\end{array}$ \\
\hline $\begin{array}{l}\text { paroxétine }(40) \text {, clonazépam }(21) \text {, temazépam }(78) \\
\text { sertraline }(<20) \\
\text { méthadone }(82) \\
\text { diazépam }(450) / \text { nordiazépam }(425) \\
\text { bromazépam }(\underline{945}) \\
\text { nordiazépam }(\underline{12200}) \text {, cetirizine } \\
\text { bromazépam }(\underline{540}) \text {, méthadone }(<20) \\
\text { paroxetine }(51) \\
\text { bromazépam }(\underline{360}) \\
\text { nordiazépam }(\underline{1415}) \\
\text { nordiazépam }(\underline{5760}) \text {, bromaz. }(\underline{885}), \text { méthad. }(\underline{220}) \\
\text { nordiazépam }(<20) \\
\text { nordiazépam }(100) \\
\text { diaz. }(\underline{910}) / \text { nordiaz. }(165) \text {, trazodone }(230) \text {, méthad. }(<20) \\
\text { zolpidem }(65) \\
\text { bromazépam }(\underline{860}) \\
\text { bromazépam }(\underline{685}) \\
\text { méthadone }(110)\end{array}$ & 0,26 & $\begin{array}{l}\text { Cnb/Amph } \\
\mathrm{Cnb} \\
\mathrm{Cnb} \\
\mathrm{Coc} \\
\mathrm{Cnb} \\
\text { Opc/Coc/Cnb } \\
\text { Opc/Coc/Cnb } \\
\mathrm{Cnb} \\
\mathrm{Cnb} \\
\mathrm{Amph} \\
\text { Opc } \\
\mathrm{Cnb} \\
\mathrm{ND} \\
\text { Opc } \\
\mathrm{Cnb} \\
\mathrm{Amph} \\
\text { Ocp/Coc } \\
\text { Cnb/Amph }\end{array}$ & $\begin{array}{l}\text { MDMA } \\
\text { THC } \\
\text { BE } \\
\text { BE, Morphine } \\
\text { BE } \\
\text { THC }\end{array}$ \\
\hline Total $=18$ & Total $=3$ & & Total $=10$ \\
\hline
\end{tabular}

\section{Tests d'urine}

Dans 12 cas, un échantillon d'urine n'a pas été fourni. Le cannabis est la substance la plus fréquemment détectée $(80 \%)$ et s'est avéré être dans $61 \%$ des échantillons le seul paramètre positif.

\section{Concentrations de drogues détectées dans le plas- ma, inférieures à la valeur limite}

Dans $39 \%$ des 133 échantillons de plasma ("faux positifs"), une ou plusieurs drogues ont été détectées en concentration inférieure à la valeur limite légale, généralement du cannabis et des amphétamines. 


\section{Présence d'alcool}

Sur les 123 échantillons de plasma ("faux positifs") soumis pour alcoolémie, $37 \%$ se sont révélés positifs, avec une majorité $(70 \%)$ d'alcoolémies supérieures à la limite légale de $0,5 \mathrm{~g} / \mathrm{l}$ (après conversion de la concentration dans le plasma par rapport à la valeur correspondante pour le sang total: limites $0,08-2,32 \mathrm{~g} / 1$; moyenne $0,82 \mathrm{~g} / \mathrm{l})$.

Une vérification ultérieure des rapports de police a démontré que tous les cas d'alcoolémie supérieure à $0,5 \mathrm{~g} / \mathrm{l}$ avaient été détectés dans l'air expiré sur place, et sanctionnés. Dans 5 cas, cette mesure correspondait à un taux d'alcoolémie dans le sang légèrement inférieure à la limite légale, tandis que dans 5 autres cas une faible alcoolémie était détectée en dépit d'un test négatif.

Dans environ la moitié $(43,5 \%)$ des alcoolémies positives, les analyses ont également mis en évidence la présence de drogues illicites en concentration inférieure à la valeur limite, généralement du cannabis et des amphétamines.

\section{Présence de substances médicamenteuses}

La présence de médicaments psychotropes a été recherchée dans 128 échantillons de plasma: 18 échantillons (14\%) contenaient entre un et trois médicaments, principalement des benzodiazépines - avec le nordiazépam et le bromazépam comme composants principaux - $(15$ cas), des antidépresseurs ( $n=5)$ et de la méthadone $(n=5)$. Dans 10 cas, les concentrations de benzodiazépine étaient supérieures à la limite thérapeutique (5). Seuls 3 échantillons montraient une combinaison d'alcool et de médicaments. Dans 10 cas, des drogues ont été trouvées à une concentration inférieure à la légale.

Dans 4 cas où le test d'urine s'est révélé positif uniquement pour la classe des opiacés, l'analyse de plasma correspondante s'est avérée négative pour la présence de morphine, mais positive pour la codéine (concentrations allant de 12 à $62 \mathrm{ng} / \mathrm{ml}$ ), souvent présent dans les analgésiques légers et les antitussifs.

\section{Aucune substance psychoactive}

Dans environ un tiers des cas "faux positifs", aucune trace d'alcool, de drogue cible ou de médicament psychoactif n'a été détectée. Dans les 37 cas où un échantillon d'urine était disponible, les tests urinaires étaient positifs pour les cannabinoïdes $(89,2 \%)$, seuls $(67,6 \%)$ ou combinés avec les amphétamines $(10,8 \%)$, les opiacés $(8,1 \%)$ ou les deux $(2,7 \%)$. Trois urines $(8,1 \%)$ étaient positives pour les amphétamines seules, un prélèvement $(2,7 \%)$ était positif aux opiacés seuls.

\section{Groupe de contrôle confirmé positif pour drogues illicites : présence d'alcool et de médicaments.}

La sélection d'un "groupe de contrôle" $(\mathrm{n}=74)$ a été basée sur la présence des différentes drogues illicites dans l'ensemble de la population soupçonnée de conduite sous l'influence de drogues : un grand groupe positif pour le cannabis, un groupe important positif pour les stimulants (amphétamines et cocaïne) et une population potentiellement intéressante positive aux opiacés. Les données détaillées concernant la présence d'alcool, de médicaments psychoactifs et de drogues illicites figurent dans les tableaux VII-VIII.

Dans 8 cas sur les 9 échantillons positifs pour l'alcool, la présence de MDMA a été détectée en combinaison avec du THC ou de la benzoylecgonine. Ce groupe de contrôle contenait également un grand nombre de personnes consommant des médicaments, usant ou abusant principalement de benzodiazépines.

\section{Discussion}

\section{La batterie de tests standardisés}

Comme une partie de la procédure (test urine, prise de sang) constitue une atteinte à des droits fondamentaux tels que l'intégrité physique et l'intimité, le législateur a voulu éviter les contrôles subjectifs, en imposant comme préalable la détection de signes extérieurs de consommation d'un des produits reconnus comme ayant une influence négative sur la conduite d'un véhicule. Dans la circulaire pour les Parquets, on mentionne que cette batterie de tests a été mise au point sur base d'analyses et d'expérience, et ensuite affinée sur bases d'applications à l'étranger. Elle permet avant tout

Tableau VII : Détail des alcoolémies positives dans le "groupe de contrôle".

\begin{tabular}{|ccc|}
\hline $\begin{array}{c}\text { Alcool }+ \\
(\mathbf{g} / \mathbf{l})\end{array}$ & Médicaments & Drogues \\
\hline 0,12 & + & THC \\
0,19 & & BE, MDMA \\
0,30 & & THC, MDMA \\
0,34 & & THC, MDMA, Amph \\
0,43 & & BE, MDMA \\
$\mathbf{0 , 5 1}$ & + & BE, MDMA, Amph \\
$\mathbf{0 , 8 9}$ & & THC, MDMA \\
$\mathbf{0 , 9 2}$ & & BE, MDMA \\
$\mathbf{0 , 9 9}$ & & BE, MDMA \\
\hline
\end{tabular}


Tableau VIII : Détail des cas positifs pour les médicaments dans le "groupe de contrôle".

\begin{tabular}{|c|c|c|}
\hline $\begin{array}{l}\text { Médicaments } \\
(\mu \mathrm{g} / \mathrm{l}) \text { (supra-thérapeutique) }\end{array}$ & $\begin{array}{l}\text { Alcool } \\
(\mathrm{g} / \mathrm{l})\end{array}$ & Drogues \\
\hline $\begin{array}{l}\text { flunitrazépam }(\underline{92}) \text {, desméthyl+amino, nordiazépam (95) } \\
\text { diazépam }(37) \text {, nordiazépam }(80) \\
\text { diazépam }(430) \text {, nordiazépam }(190) \\
\text { bromazépam }(\underline{3100}) \\
\text { bromazépam }(\underline{380}) \text {, méthadone }(180) \\
\text { bromazépam }(\underline{540}) \text {, tétrazépam }(36) \text {, méthadone (135) } \\
\text { nordiazépam }(280) \text {, méthadone }(340) \\
\text { diaz. }(75) \text {, nordiaz. }(\underline{1500}) \text {, bromaz. }(90) \text {, alprazolam }(47) \\
\text { méthadone }(195) \text {, trazodone }(300) \\
\text { diazépam }(80) \text {, nordiazépam }(60) \\
\text { bromaz. }(32) \text {, diazépam }(\underline{900}) \text {, nordiaz. (600), lormétaz. (47) } \\
\text { bromazépam }(\underline{1100}) \\
\text { bromazépam }(\underline{415}) \text {, nordiazépam }(\underline{3200}) \text {, tétrazépam (185) } \\
\text { diazépam }(420) \text {, nordiazépam }(500)\end{array}$ & 0,12 & $\begin{array}{l}\text { THC } \\
\text { THC, Amph, MDMA } \\
\text { BE, Amph, MDMA } \\
\text { Morph } \\
\text { Morph, BE } \\
\text { Morph } \\
\text { Morph, BE } \\
\text { BE, MDMA } \\
\text { Morph, BE } \\
\text { THC } \\
\text { Morph, THC } \\
\text { THC } \\
\text { Morph, BE, THC }\end{array}$ \\
\hline Total $=13$ & Total $=2$ & \\
\hline
\end{tabular}

de détecter l'influence réelle et ainsi limiter au strict minimum le nombre de faux positifs. Toutefois il convient de préciser qu'une condition essentielle est que cette méthode doit être appliquée par des policiers qui ont été formés spécifiquement, et entretiennent une expérience en la matière.

Chaque individu est différent et peut réagir autrement après consommation de stupéfiants. Personne ne peut: s'assurer que l'individu présentera toujours les mêmes signes ou le même comportement. La batterie de tests comporte la majorité des signes possibles. Néanmoins, il faudra toujours faire preuve de bon sens et tenir compte des circonstances. Des facteurs externes peuvent influencer la performance (personne âgée, nerveuse, fatiguée, les circonstances climatiques,

Appliquer la batterie de tests de façon mathématique selon la règle minimale d'un signe dans la première partie et un signe dans la partie tests de répartition d'attention ne va pas conduire à chaque fois à des positifs au test urine ou à la prise de sang. Le policier bien formé et expérimenté, faisant preuve de bon sens, aura plus de facilité à détecter un conducteur sous influence, ceci étant le but de cette loi.

Après la sélection par une batterie de tests, $78.1 \%$ des tests urinaires étaient positifs pour le cannabis, $49.6 \%$ pour les amphétamines dans $49.6 \%, 20.2 \%$ pour les métabolites de la cocaïne et $9.5 \%$ pour les opiacés. Ces pourcentages sont fortement influencés par la sélection des lieux de contrôles (voir plus loin 4.3).

Certains tribunaux locaux ont informé leurs services de police que lorsque l'analyse de l'air expiré se révèle positive, ils préfèrent poursuivre le conducteur pour conduite en état d'ivresse plutôt que de continuer les tests pour déceler la présence éventuelle de drogues, tests qui sont beaucoup plus coûteux et fastidieux. Une circulaire nationale demande néanmoins aux services de police d'effectuer les deux procédures, et il est hautement recommandé d'harmoniser la stratégie dans ce contexte.

\section{Les "faux positifs"}

Le bien-fondé de la nouvelle procédure légale destinée à détecter les conducteurs roulant sous l'influence de drogues illicites est prouvé par le nombre d'analyses de plasma confirmées positives. Etant donné que la proportion de cas non établis $(15 \%)$ nous semblait relativement élevée, nous avons examiné les raisons pour lesquelles la batterie de tests standardisés pouvait avoir un résultat positif ou les raisons pour lesquelles un test d'urine positif ne pouvait pas être confirmé par la présence dans le sang de drogues illicites en concentration supérieure à celle autorisée.

- Dans $39 \%$ de ces cas, les concentrations de drogues illicites détectées se sont avérées inférieures à la valeur limite. Le fait de raccourcir le délai avant la prise d'un échantillon de sang pourrait augmenter le nombre de tests positifs. De plus, étant donné que certains analytes comme le THC et la cocaïne sont instables dans des matrices biologiques, il convient de prendre certaines précautions pour la conservation et le transport des échantillons de sang. Un autre point de discussion est la suppression éventuelle des valeurs limites légales pour la présence de drogues dans le plasma, ce qui mènerait à une véritable politique de "tolérance zéro", 
à l'instar de l'exemple allemand et suédois $(6,7)$. En tout cas, des schémas de contrôle de qualité doivent garantir la cohérence des résultats entre les différents laboratoires $(8,9)$. En Belgique l'agrément d'un laboratoire en vue du dosage de stupéfiants dans le cadre de la conduite automobile est basée sur une participation au programme de qualité allemand ( 3 enquêtes par an ; un minimum de 2 résultats acceptés pour chaque composé stipulé dans la loi) (9).

- Environ un quart de la population étudiée ("faux positifs") montrait une alcoolémie supérieure à la limite légale de $0,5 \mathrm{~g} / \mathrm{l}$. Ces conducteurs avaient été soumis à un dépistage dans l'air expiré sur le terrain, et sanctionnés en conséquence (interdiction de conduire pendant 6 heures + amende). Il est clair que dans ces cas, l'incapacité à conduire est principalement due à l'influence de l'alcool. On peut cependant imaginer qu'une alcoolémie faible combinée à une concentration réduite de drogues illicites ( 5 cas) peut amoindrir la capacité à conduire jusqu'à un degré détectable par le test de sobriété effectué sur place.

- Les résultats des analyses des médicaments montrent que $14 \%$ de ces conducteurs contrôlés avaient consommé des médicaments psychoactifs - les benzodiazépines étant particulièrement populaires - en combinaison avec des drogues illicites ou pas. A l'exception d'un seul conducteur, la consommation de médicaments n'a pas été accompagnée de consommation d'alcool, ou alors à une concentration faible. L'impact de la consommation de benzodiazépines sur le comportement au volant a été démontré dans de nombreuses études (10). La présence de concentrations supra-thérapeutiques et toxiques étant probablement responsable de l'incapacité détectée, c'est un argument valable pour demander l'introduction des médicaments psychoactifs - et en premier lieu les benzodiazépines - dans la loi portant sur le code de la route, comme cela a récemment été fait en Suède en cas de dose supra- thérapeutique ou d'utilisation sans ordonnance médicale (7). Utiliser un seuil universel est impossible compte tenu des concentrations thérapeutiques et toxiques très variées. En plus, des réactions croisées différentes dans les tests immunochimiques compliquent la détection urinaire. Néanmoins, une conduite inadéquate combinée avec un abus de médicaments prouvé devrait être punissable.

- Enfin, pour le dernier tiers des sujets étudiés, aucune trace d'alcool, de drogues cibles ou de médicaments psychoactifs n'a été détectée dans le plasma. Une autre explication possible de la diminution de leur capacité à conduire peut être : la présence d'autres drogues non spécifiées dans la loi (par ex. GHB, LSD), des condi- tions physiologiques telles que la maladie ou la fatigue, ou encore la mauvaise exécution des tests sur le terrain, liée au manque d'expérience du fonctionnaire de police concerné.

Nos résultats sont comparables aux résultats observés en Suède, où $82 \%$ des échantillons étaient positifs pour des drogues ou des médicaments, dans $6 \%$ des cas seul l'alcool était retrouvé et dans $12 \%$ ni drogues, ni médicaments, ni alcool ne furent retrouvés (11).

\section{"Groupe de contrôle"}

Un grand nombre de cas 'uniquement THC' ont été détectés lors de contrôles destinés aux personnes fréquentant les "coffee-shops". Comme on pouvait s'y attendre, aucun de ces conducteurs ne s'est avéré positif pour l'alcool car ces endroits n'ont pas de licence de vente de boissons alcoolisées. En revanche, les visiteurs de ce que l'on appelle les "after-clubs", consommateurs fréquents de cocaine et d'autres stimulants, combinent ces drogues avec de l'alcool.

La présence de médicaments est surtout retrouvée chez les hérö̈nomanes (dans $50 \%$ des cas où des médicaments ont été retrouvés, la morphine était positive).

\section{Les cas positifs pour le cannabis}

La distribution des concentrations retrouvées dans les plasmas uniquement positifs pour le THC est présentée pour les années 2000-2001 de l'étude décrite, complétée avec les données de 2002 et 2003 (premier trimestre) (Fig. 4). Une concentration de THC entre 1 et $2 \mathrm{ng} / \mathrm{ml}$ est retrouvée dans environ $10 \%$ des cas. L'élimination rapide de THC du plasma en fonction du temps écoulé après la consommation du cannabis, et l'effet pharmacodynamique continuant même quand les taux plasmatiques approchent la limite de détection de la méthode analytique sont des arguments valables pour diminuer la limite autorisée pour le THC.

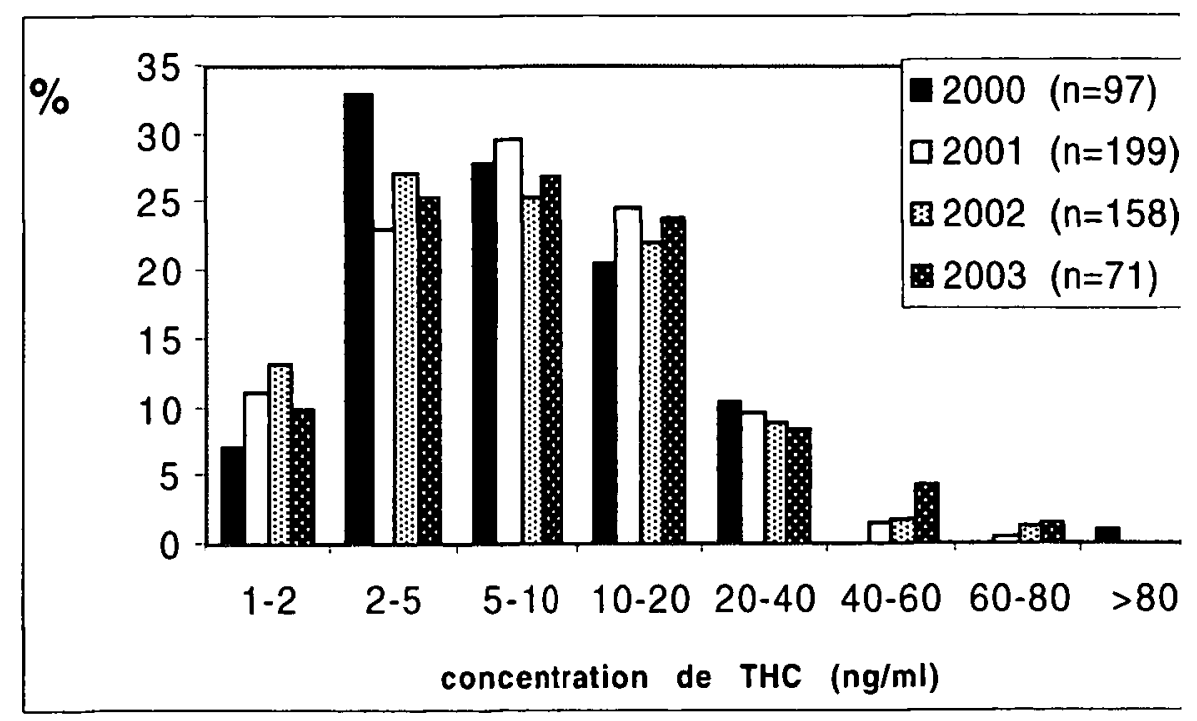

Figure 4 : Concentrations de THC observées dans les échantillons de plasma uniquement positifs pour le THC. 


\section{Conclusion}

En général les résultats démontrent que dans ses premières années d'application la nouvelle procédure légale a été menée à bon terme, c'est à dire une pénalisation pour conduite sous influence de stupéfiants dans $85 \%$ des échantillons analysés. Dans la population présentée, l'absence de valeurs limites légales aurait résulté en une diminution des "faux-positifs" de $15 \%$ à $9 \%$. Tenant compte des cas légalement positifs pour l'alcool, ce nombre descend à $7 \%$. Des médicaments ont été retrouvés dans $14 \%$ des cas "faux positifs", souvent (55\%) en combinaison avec des concentrations de drogues illicites sous le seuil légal.

Suite à ces actions policières de contrôle de conduite sous influence de stupéfiants les policiers nous rapportent un changement de comportement des jeunes : pendant leurs sorties un chauffeur se sacrifie et reste sobre, tandis que les passagers de sa voiture consomment l'alcool et/ou des stupéfiants à volonté ; un comportement qui devrait résulter en une fréquence diminuée d'accidents routiers.

\section{Remerciements}

Les auteurs souhaitent exprimer leurs remerciements particuliers à B. Laeremans et à A. Coppe pour leur aide et leur participation à la collecte, au traitement et à l'interprétation des données.

\section{Références}

1. Verstraete A., Maes V. The elaboration of the new 'per se' legislation on drugs and driving in Belgium. In: Laurel H. ed. T2000 Alcohol, drugs and traffic safety, Proceedings of the 15th International Conference, Stockholm, Sweden, 2000, CD-ROM paper 545.

2. Samyn N., Viaene B., Laeremans B., De Boeck G. Evaluation of different roadside drug testing equipment. Contribution of Belgium. ROSITA D4, DG VII PL983032, 1999, (http://www.rosita.org).

3. Samyn N., De Boeck G., Verstraete A. The use of oral fluid and sweat wipes for the detection of drugs of abuse in drivers. J. Forensic Sci. $2002 ; 47$ (6) : 1380-7.

4. Pounder D.J., Jones A.W. Measuring blood-alcohol concentration for clinical and forensic purposes. In: Karch S. ed. Drug Abuse Handbook, Boca Raton : CRC Press, 1998, 356-373.

5. Uges D. List of determinations for clinical toxicology and therapeutic drug monitoring. Dept. of Pharmacy, University Hospital, Groningen, The Netherlands, 1998. Version mise à jour: http://www.tiaft.org (accessible aux members TIAFT).
6. Steinmeyer S., Ohr H., Maurer H.J., Moeller M.R. Practical aspects of roadside tests for administrative traffic offences in Germany. Forensic Sci. Int. $2001 ; 121$ : 33-6.

7. Ceder G. Drugged driving in Sweden - effects of new legislation concerning zero-tolerance for narcotic drugs. In: Laurel H. ed. T2000 Alcohol, drugs and traffic safety, Proceedings of the 15th International Conference, Stockholm, Sweden, 2000, CD-ROM paper 509.

8. Société Française de Toxicologie Analytique (SFTA, France), commission assurance de qualité, http://www.sfta.org

9. Society of Toxicological and Forensic Chemistry (GTFCh, Germany), quality assurance committee, http://www.gtfch.org

10. Maes V., Charlier C., Grenez O., Verstraete A. Drugs and medicines that are suspected to have a detrimental impact on road user performance. ROSITA D1, DG VII PL983032, 1999, (http://www.rosita.org).

11. Ahlner J., Hommgren P. Drugs and driving in Sweden in 2001 - experience from a new legislation. Ann. Toxicol. Anal. 2002; 14(3) : 223. 